\title{
Evaluation of the ablation margin of hepatocellular carcinoma using CEUS-CT/ MR image fusion in a phantom model and in patients
}

\author{
Kai Li ${ }^{\dagger}$, Zhongzhen Su ${ }^{\dagger}$ Erjiao Xu, Qiannan Huang, Qingjing Zeng and Rongqin Zheng*
}

\begin{abstract}
Background: To assess the accuracy of contrast-enhanced ultrasound (CEUS)-CT/MR image fusion in evaluating the radiofrequency ablative margin (AM) of hepatocellular carcinoma (HCC) based on a custom-made phantom model and in HCC patients.

Methods: Twenty-four phantoms were randomly divided into a complete ablation group $(n=6)$ and an incomplete ablation group $(n=18)$. After radiofrequency ablation (RFA), the AM was evaluated using ultrasound (US)-CT image fusion, and the results were compared with the AM results that were directly measured in a gross specimen.

CEUS-CT/MR image fusion and CT-CT / MR-MR image fusion were used to evaluate the AM in 37 tumors from 33 HCC patients who underwent RFA.

Results: The sensitivity, specificity, and accuracy of US-CT image fusion for evaluating AM in the phantom model were 93.8, 85.7 and 91.3\%, respectively. The maximal thicknesses of the residual AM were $3.5 \pm 2.0 \mathrm{~mm}$ and $3.2 \pm 2.0 \mathrm{~mm}$ in the US-CT image fusion and gross specimen, respectively. No significant difference was observed between the US-CT image fusion and direct measurements of the AM of HCC. In the clinical study, the success rate of the AM evaluation was 100\% for both CEUS-CT/MR and CT-CT/MR-MR, and the duration was $8.5 \pm 2.8 \mathrm{~min}$ (range: 4-12 min) and $13.5 \pm 4.5 \mathrm{~min}$ (range: $8-16 \mathrm{~min}$ ) for CEUS-CT/MR and CT-CT/MR-MR, respectively. The sensitivity, specificity, and accuracy of CEUS-CT/MR imaging for evaluating the AM were 100.0, 80.0, and 90.0\%, respectively.

Conclusions: A phantom model composed of carrageenan gel and additives was suitable for the evaluation of HCC AM. CEUS-CT/MR image fusion can be used to evaluate HCC AM with high accuracy.
\end{abstract}

Keywords: Tumor ablation, Phantom model, CEUS, CT, Image fusion

\section{Background}

Radiofrequency ablation (RFA) is a radical treatment for hepatocellular carcinoma (HCC) and has a relatively low risk $[1,2]$. However, recent studies have shown that HCC patients undergoing RFA have a higher rate of local tumor progression (LTP) compared with HCC patients treated with resection [3-6]. Independent factors associated with LTP include tumor size, sub-capsular

\footnotetext{
* Correspondence: zhengrongqin345@sina.com

${ }^{\dagger}$ Equal contributors

Department of Ultrasound, The Third Affiliated Hospital of Sun Yat-sen University, Guangzhou 510630, Guangdong Province, People's Republic of China
}

location, blood vessel proximity, and an insufficient ablation margin (AM) [7-15]. The term "ablative margin" refers to the 0.5 to 1.0 -cm-wide region of normal tissue around the tumor that should ideally be removed during tumor ablation [16, 17]. Therefore, AM is one of the most important factors for the prediction of LTP in HCC patients after RFA [18-20]. However, regular medical imaging methods, including CT, MR, and contrastenhanced ultrasound (CEUS), are not able to accurately evaluate AM because the tumor and surrounding normal liver tissue mix and merge in the ablative area, and the boundary between normal tissue and the ablative area is difficult to identify. Thus, using the current 
imaging methods, it is challenging to determine whether the zone of ablation encompasses the range of the AM around the index tumor.

In recent years, novel medical imaging methods have been explored to assess AM in HCC patients after ablation, including CT-CT image fusion [21, 22], MR-MR image fusion [23, 24], contrast-enhanced ultrasound (CEUS)-CT/MR image fusion $[18,25]$, MR with impaired clearance of ferucarbotran [26, 27], and MR with gadolinium ethoxybenzyl diethylene triamine pentaacetic acid [28]. Our group has reported that CEUS-CT/MR image fusion, which can be applied intraoperatively, is useful for assessing AM in HCC patients receiving ablation [25]. An accurate evaluation of AM based on CEUS-CT/MR image fusion allows physicians to perform supplementary ablation, increasing the number of adequate AM and reducing the probability of LTP. However, direct measurement of AM in HCC patients is not feasible because the gross specimen is usually unavailable in ablation patients. Therefore, the rate of LTP has been widely used as the standard to evaluate the accuracy of AM in most studies. Tumor tissues that are not covered by AM during $\mathrm{HCC}$ ablation are usually a major cause of LTP. However, AM is not the only independent factor associated with LTP after ablation. Therefore, the accuracy of CEUS-CT/MR image fusion in assessing AM should be further evaluated.

In this study, we established a phantom model to evaluate AM based on US-CT image fusion. The aim of this study was to assess the accuracy of CEUS-CT/MR image fusion for the evaluation of the AM of liver tumors, both in an in vitro phantom model and in the clinic. The gross specimen of the phantom after RFA was used as a gold standard. The clinical AM results obtained using CEUS-CT/MR image fusion and MR-MR/ CT-CT image fusion were compared.

\section{Methods}

\section{Materials}

The materials used to construct the phantom model included carrageenan (Dehui Marine Biological Technology Cor., Ltd., Qingdao, China) and a number of additives such as oral ultrasonic contrast agent (Hangzhou Huqingyutang Medical Technology Cor., Ltd., Hangzhou, China), gastric window contrast agent (Huqingyutang Cor., Hangzhou, China), milk, and Congo red.

\section{Establishment of the phantom model}

The phantom model included a spherical tumor model (2 cm in diameter, Fig. 1a), an AM model (a 5-mm layer of AM gel around the tumor model, Fig. 1b), and a cylindrical parenchyma model $(10 \mathrm{~cm}$ in height and diameter, Fig. 1c), in which the AM model was embedded (Fig. 1d). Bamboo sticks in the parenchyma model were used as registration and positional marks.

\section{Model testing}

The shape, height, and gradient of the phantom model were tested at $1 \mathrm{~h}, 6 \mathrm{~h}$, and $12 \mathrm{~h}$ after construction of the model. The structure of the phantom model and the position of the marks were checked using both ultrasound and observation of the gross specimen.

\section{Study grouping}

A total of 24 phantom models were randomly divided into two groups, including a complete ablation group $(n=6)$ in which the ablative area was covered by AM and an incomplete ablation group $(n=18)$ in which the ablative area was not covered by AM.

\section{Radiofrequency ablation}

RFA was performed with a cooled-tip RFA system (Covidien, Mansfield, MA, USA) using a 17-gauge,

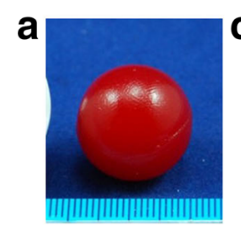

b
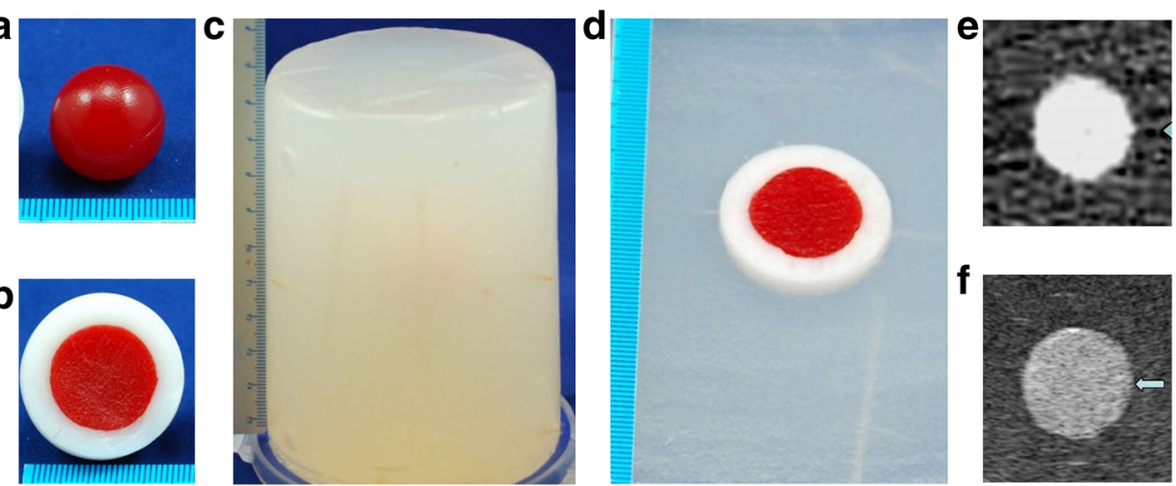

Fig. 1 a A spherical tumor model ( $2 \mathrm{~cm}$ in diameter) made of carrageenan in red. $\mathbf{b}$ Section of the AM model: the carrageenan tumor model surrounded by the 5-mm AM gel in white. c Cylindrical-shaped parenchyma model $(10 \mathrm{~cm}$ in height and $10 \mathrm{~cm}$ in diameter of the upper and lower plane). $\mathbf{d}$ Section of the cylindrical-shaped parenchyma model. e CT image showing the tumor. The scope of AM could not accurately evaluated. $\mathbf{f}$ US image showing the tumor. The boundary between the tumor and AM gel was not clear 
internally cooled-tip electrode with a 3-cm tip. A MyLab Twice ultrasound machine (Esoate, Genoa, Italy) and a linear probe LA332 (frequency range from 3 to $11 \mathrm{MHz}$ ) with imaging fusion (Virtual Navigation System) and three-dimensional software were employed for ultrasound guidance and exploration.

An ablative area model was established by ablating the tumor model. Electrodes were inserted into the cylindrical model parenchyma via ultrasound guidance by an experienced ultrasound interventional doctor. The RFA was set in impedance mode with maximum output. According to our pilot studies, the duration of ablation was 4 and $6 \mathrm{~min}$ for the incomplete and complete ablation groups, respectively. After ablation, the liver tumor model together with the AM model and a portion of the parenchyma model were melted and mixed. An ablative area model was established after cooling and solidifying the melted gel.

\section{Evaluation of AM by US-CT image fusion}

Each phantom CT scan was performed without contrast medium prior to ablation. The CT scan was performed using a 64-row multi-detector CT scanner (VCT 64 slices; GE Medical Systems). The following CT parameters were applied to acquire dynamic data: 1-s gantry rotation time, $120 \mathrm{kV}, 80 \mathrm{~mA}$, acquisition in 264 transverse mode (64 sections per gantry rotation), and 2.5-mm reconstructed section thickness (Fig. 2A-1, B-1). The model was positioned horizontally with the guidance of a horizontal laser instrument.

Image fusion was performed by an experienced ultrasound doctor who was blinded to the ablation. A series of CT data in DICOM format were uploaded in fusion mode into the ultrasound system to automatically generate images. The areas of the tumor model and 5-mm AM in the 3D CT images were outlined using red and blue circles, respectively. At the beginning of image fusion, the

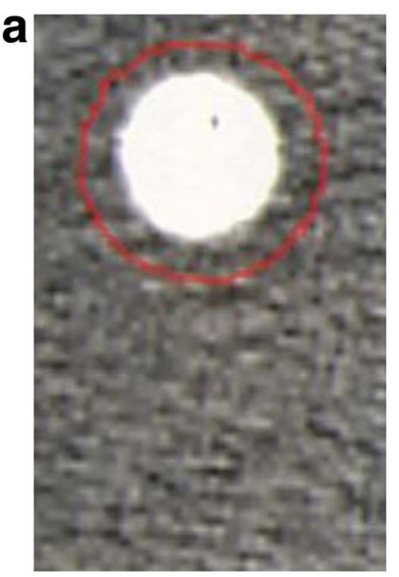

A-1

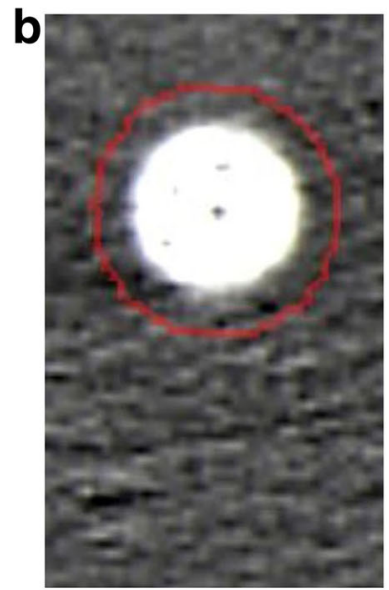

B-1

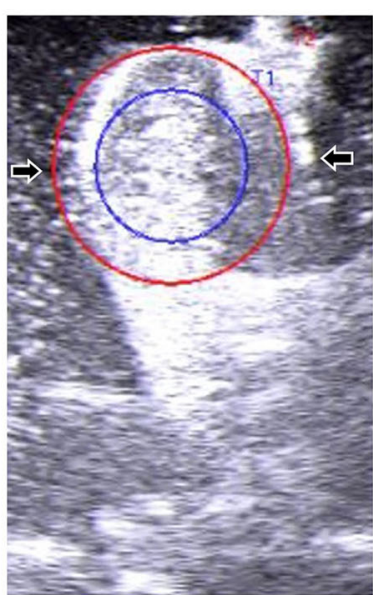

A-2

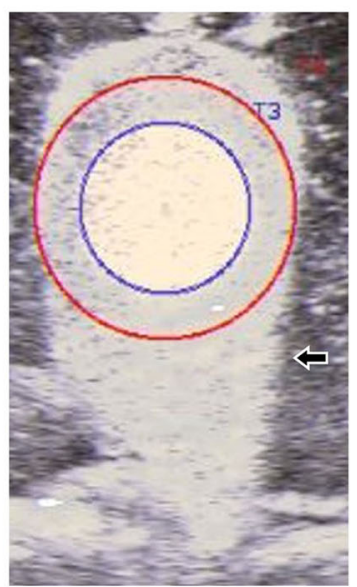

B-2

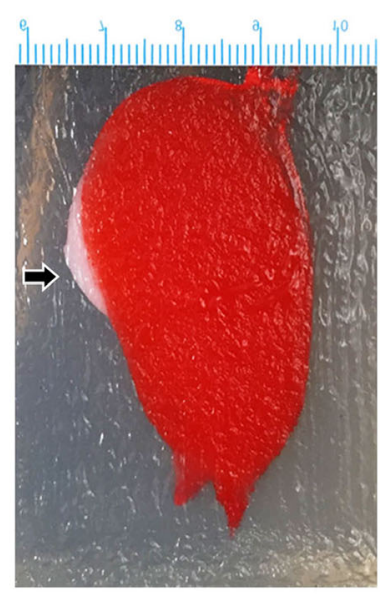

A-3

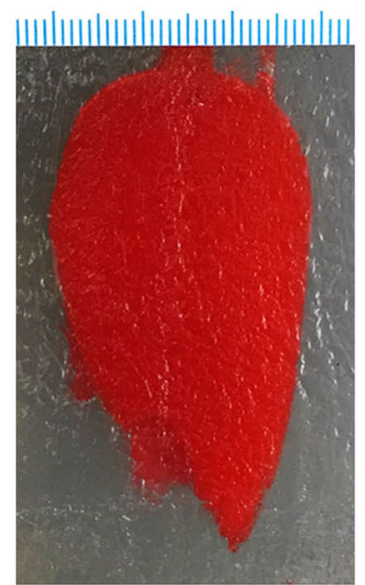

B-3

Fig. 2 (A-1) and (B-1): CT images of the models. (A-2) and (B-2): US-CT image fusion of the area of ablation (left arrow). The AM was not fully encompassed by the area of ablation (right arrow, A-2). The tumor and AM were completely encompassed by the area of ablation (B-2). (A-3): The AM was not completely encompassed by the area of ablation in the gross specimen (left arrow). (B-3): The AM was completely encompassed by the area of ablation in the gross specimen. The tumor is shown in blue, and the AM gel is indicated in red ( $5 \mathrm{~mm}$ ) 
registration marks in the phantom models were used to choose one transverse section of the CT image and the ultrasound image of the same section. The CT and ultrasound images were then overlapped and fused. After registration of this section, additional fine tuning was performed to enable a more precise adaptation. The distance between the CT and ultrasound images of the same registration mark in overlapping mode could be measured and used as the error of image fusion. A successful image fusion was defined when the error of image fusion of all registration marks was less than $2 \mathrm{~mm}$. Otherwise, the registration was repeated. The image fusion was considered a failure if a successful fusion could not be achieved after three attempts. In overlapping mode, inclusion of the tumor within the ablative area of the model and the AM mode could be decided. The position and thickness of the thickest part of the AM model was recorded if the AM was not completely covered (Fig. 2A-2, B-2).

\section{Evaluation of the AM in the gross specimen}

The phantom was cut along the section showing the thickest residue in the AM model by image fusion. Whether the AM model had been fully ablated was examined, and the maximal thickness of the AM model residue was measured. In addition, the results of the US-CT image fusion and gross specimen were compared to calculate the sensitivity, specificity, and accuracy of US-CT image fusion for the evaluation of AM model residue (Fig. 2A-3, B-3).

\section{Ethics statement and study populations}

This study was approved by the Institute Research Medical Ethics Committee of the Third Affiliated Hospital of Sun Yat-Sen University and was in compliance with the Declaration of Helsinki. Informed consent was obtained from all participants. From January 2014 to April 2014, a total of 33 HCC patients who underwent RFA in our hospital were enrolled in this study. All liver lesions meeting the Milan criteria were pathologically or clinically diagnosed as HCC [29]. Inclusion criteria were as follows: the ablation zone of the tumors was evaluated by CEUS-CT/MR image fusion after RFA. Exclusion criteria were as follows: 1) failure to obtain CT/MR data in DICOM format from the patient preoperatively; 2) the patient did not receive a CT/MR examination 1-2 months after RFA; 3) different image methods (CT and MR) were applied preoperatively and postoperatively, precluding image fusion; 4) ultrasound and CT/MR images could not be successfully fused; 5) the patient was allergic to ultrasound contrast agents.

\section{RFA}

We used the same cooled-tip RFA system in the phantom model research for HCC patient RFA. The ablation was performed under endotracheal anesthesia. All RFA procedures were performed by two experienced ultrasound physicians with more than 5 years of RFA experience. According to the routine examination, previously determined plan, and multiple needle ablations for larger tumors, all $\mathrm{HCC}$ lesions, including the 5-mm AM, were successfully ablated. CEUS-CT/MR image fusion was performed approximately $10 \mathrm{~min}$ after RFA to evaluate the efficacy of RFA and to guide the supplementary ablation.

\section{CEUS-CT/MR image fusion}

CEUS-CT/MR image fusion was performed using the MyLab Twice (Esaote, Italy) ultrasound unit and convex array transducer CA431 (4-10 MHz) 10-15 min after ablation. Virtual Navigator was the image fusion program and CnTI $(\mathrm{MI}<0.05)$ was the imaging technique for contrast-enhanced ultrasound in the ultrasound unit. SonoVue (Bracco, Italy) was used as the contrast agent. For each application, $2.4 \mathrm{ml}$ of SonoVue was administered through the antecubital vein and flushed by $5 \mathrm{ml}$ of normal saline.

The method of CEUS- CT/MR image fusion used in this study had been reported in our former article [18]. The CT/MR image series in DICOM format were transferred into the navigation system and 3D image volume was generated. Different colors were used to outline the tumor and 5-mm AM (Figs. 3A-2, 4A-2). After planar registration, more precise fusion was acquired through additional refinement. Then CEUS was performed and the image of CEUS was overlapped with the CT/MR image to see whether the area of CEUS had covered the tumor as well as the AM region.

\section{CT-CT/MR-MR image fusion}

Contrast-enhanced CT/MR was performed 1 month before and after the RFA for all patients. AM was further evaluated using CT-CT/MR-MR image fusion if the CT/ MR at 1 month after RFA revealed that the lesion was completely ablated.

One CT/MR portal or delayed phase series with a clearly demonstrated hepatic vessel and ablative area before RFA in DICOM format was transferred into the navigation system in MyLab Twice. One month after RFA, another series of CT/MR images were also imported into the image fusion system. The system then automatically displayed six pictures in two rows: the upper row included the transaction, coronal and vertical section CT/MR images before RFA, and the lower row showed the corresponding CT/MR images after RFA. The HCC lesion in the CT/MR before RFA was manually outlined, and then a 5-mm AM was set automatically in different colors (Figs. 3b, 4b). 

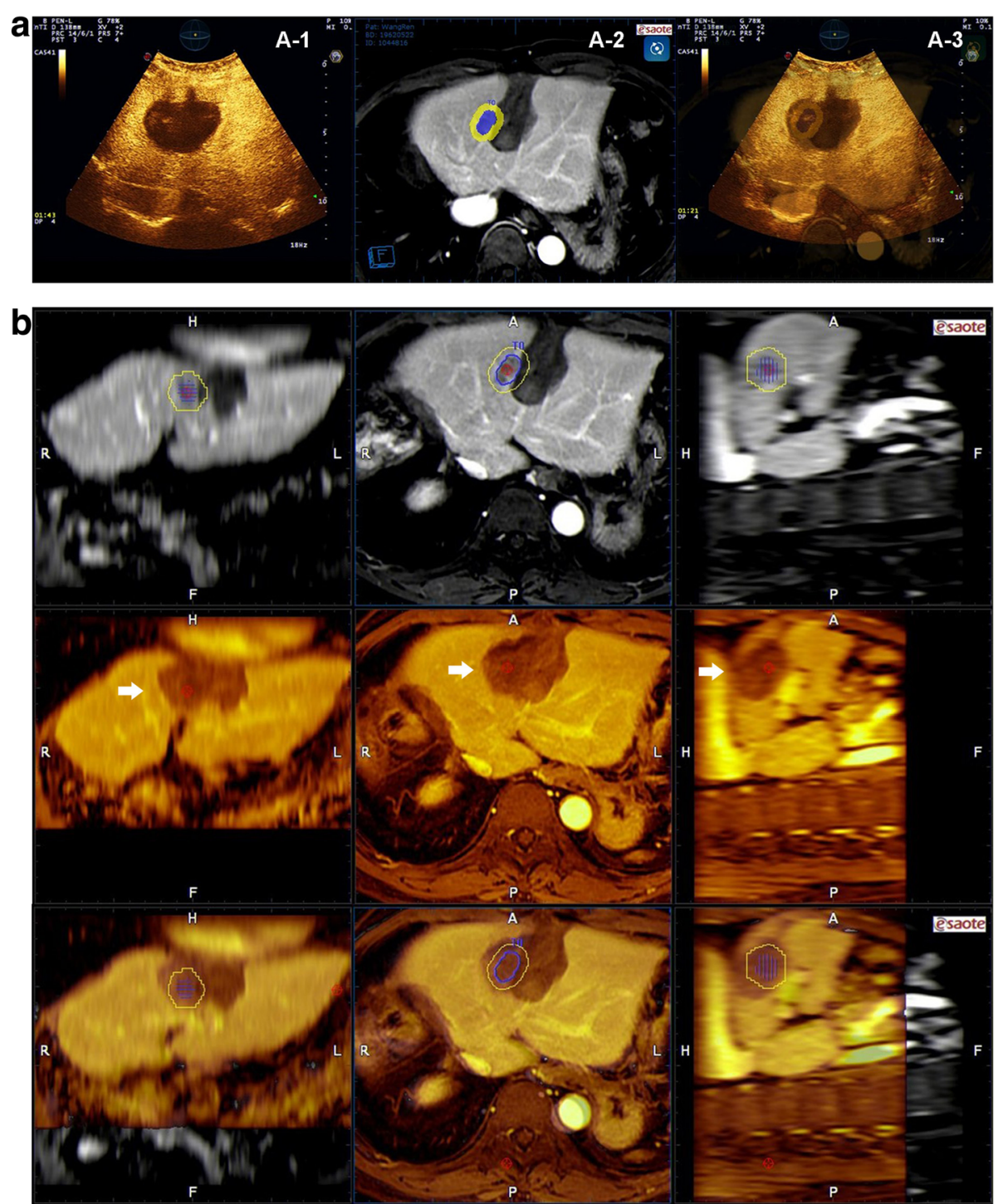

Fig. 3 Medical images of case 1 in the clinical study. (A-1) CUES image of the area of ablation (dark). (A-2). Preoperative MR image (tumor shown in blue, and 5-mm AM shown in yellow). (A-3) CEUS-MR fusion image. The tumor and 5-mm AM were fully encompassed by the area of ablation. b MR-MR fusion image of case 1. Upper panel: preoperative MR images (tumor shown in blue, and 5-mm AM shown in yellow). Middle panel: postoperative MR images (arrow, ablation area shown in dark color). Lower panel: MR-MR fusion images showing that the tumor and the 5-mm AM were fully encompassed by the area of ablation

Image registration was performed by aligning two overlaid CT/MR images. Translation and rotation were performed in three reformed planes to maximize the image similarity around the HCC lesion and the area of ablation. The hepatic vein, hepatic artery portal complex and hepatic contour near the lesion were used as landmarks for fine adjustments to obtain a satisfactory registration. The pre- and post-RFA CT/MR images were then overlapped to assess whether the ablative area encompassed the HCC lesion and the 5-mm AM. The standards of complete registration included complete matching of three corresponding anatomic landmarks adjacent to the tumor, and the offset was less than $5 \mathrm{~mm}$ in each plane. Failed registration was determined when the above standards were not achieved after three attempts. The time spent on registration for each lesion and the success rate of MRMR image fusion were recorded. The results of the CTCT/MR-MR image fusion as standard were used to evaluate the accuracy of the CEUS-CT/MR image fusion.

\section{Data analysis}

The analyzed data included 1) the duration required for the US-CT image fusion (phantom model study), CEUS$\mathrm{CT} / \mathrm{MR}$ image fusion, and MR-MR image fusion (clinical study); 2) the success rate of US-CT image fusion (phantom model study), CEUS-CT/MR image fusion and CT- 

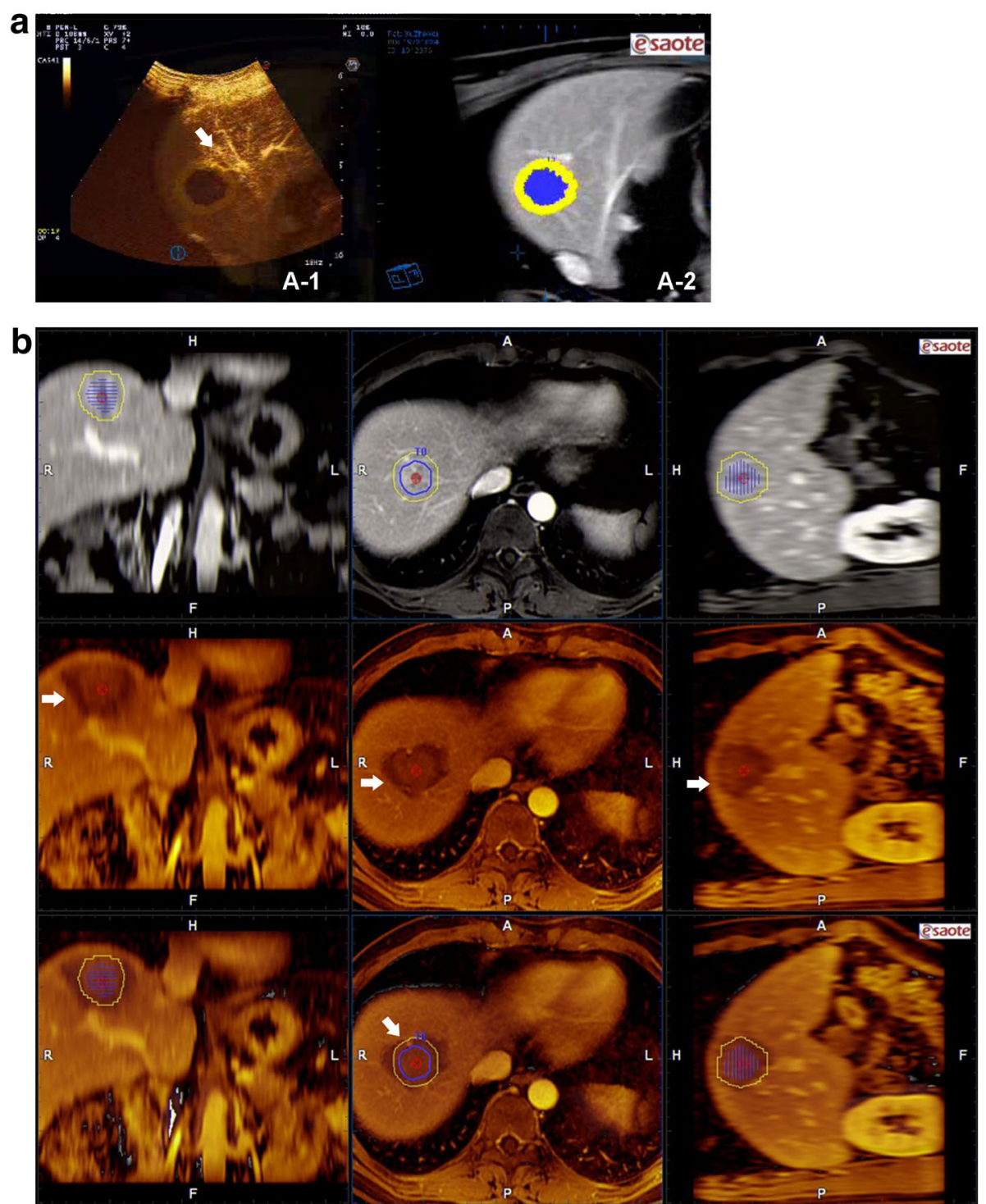

Fig. 4 Medical images of case 2 in the clinical study. (A-2) Preoperative MR image (tumor shown in blue and 5-mm AM in yellow). (A-1) CEUS-MR fusion image showing that the area of ablation encompassed the tumor but not the entire AM due to the influence of vessels (white arrow). b MR-MR fusion image from the same patient. Upper panel: preoperative MR images (tumor shown in blue and 5-mm AM in yellow). Middle panel: postoperative MR images (arrow, area of ablation shown in dark color). Lower panel: MR-MR fusion images showing that the tumor and AM were not fully encompassed by the area of ablation (white arrow)

CT/MR-MR image fusion (clinical study); 3) the accuracy rate of the assessment, including the coincidence rates of the assessment of complete ablation between US-CT image fusion and the gross specimen (phantom model study), and between CEUS-CT/MR image fusion and CT-CT/MR-MR image fusion; and 4) the maximum thickness of the residual AM in the US-CT image fusion and the gross specimen.

\section{Statistical analyses}

Statistical analyses were performed using SPSS for Microsoft Windows (version 13.0; SPSS Inc. Chicago, IL, USA). The data are the mean \pm standard deviation (range). The paired $t$ test was used to compare the maximum thickness of the residual AM in the US-CT image fusion and the gross specimen. A $P$ value less than 0.05 was considered significant.

\section{Results}

Successful establishment of the phantom models

The echogenicity, density, and color of different components of the phantom models met the requirements (Table 1). The appearance, height, and gradient of the phantom models were stable at $1 \mathrm{~h}, 6 \mathrm{~h}$, and $12 \mathrm{~h}$ after model production. 
Table 1 The ultrasound echo, CT density and color of the phantom models

\begin{tabular}{llll}
\hline Model & Ultrasound echo & CT density & Color \\
\hline Parenchyma model & Hypoechoic $^{a}$ & Low density $^{b}$ & Colorless semitransparent \\
Tumor model & Hyperechoic & High density & Red opaque \\
AM model & Hypoechoic & Milky opaque \\
Ablative area model & Slightly hyperechoic & Low density & Carnation semitransparent
\end{tabular}

${ }^{a}$ These two had the same echogenecity, ${ }^{b}$ these two had the same density

\section{US-CT image fusion detected residual AM with high sensitivity, specificity, and accuracy in the phantom models}

Of the 24 phantom models, one phantom was accidentally damaged, and 23 phantoms were used in all followup experiments. Image fusion was successfully obtained from the 23 phantoms. The success rate of image fusion was $100 \%(23 / 23)$. The average time used for image fusion was 5-12 min (median $=7 \mathrm{~min}$ ). Compared with the gross specimen, the sensitivity, specificity, and accuracy of the US-CT image fusion for the detection of residual AM were 100.0, 93.8 and 95.7\%, respectively (Table 2). In one case, the US-CT image fusion showed that AM was completely encompassed by the ablative area, but a 1-mm residual AM was still observed in the gross specimen. The maximal thicknesses of residual AM calculated by the US-CT image fusion and measured in the gross specimen were $3.5 \pm 2.0 \mathrm{~mm}$ and $3.2 \pm 2.0 \mathrm{~mm}$, respectively, which suggested that there was no significant difference $(P=0.705)$.

\section{CEUS-CT/MR image fusion revealed residual $A M$ with a high sensitivity, specificity, and accuracy in the clinical study}

A total of 30 tumors from 26 patients were enrolled in the clinical study. The clinical characteristics of the participants and HCC lesions are shown in Table 3. Seven patients were excluded from the study, including three patients without a postoperative CT/MR examination and three patients with inconsistent preoperative and postoperative imaging methods. In addition, one patient was excluded from the clinical study due to the

Table 2 The results of AM evaluated by US-CT image fusion and gross specimen $(P>0.05)$

\begin{tabular}{lllll}
\hline & & \multicolumn{2}{l}{ Gross specimen } & Total \\
\cline { 3 - 4 } & & $\begin{array}{l}\text { AM } \\
\text { covered }\end{array}$ & $\begin{array}{l}\text { AM not } \\
\text { covered }\end{array}$ & \\
\hline US-CT image fusion & AM covered & 7 & 1 & 8 \\
& AM not covered & 0 & 15 & 15 \\
& Total & 7 & 16 & 23 \\
\hline
\end{tabular}

formation of a local abscess in the ablation zone after RFA, which could bias the AM assessment.

CT-CT image fusion was conducted for one lesion, and MR-MR image fusion was applied for the remaining lesions. The success rate of CEUS-CT/MR image fusion and CT-CT/MR-MR image fusion were both 100\% (30/30). The duration was $8.5 \pm 2.8 \mathrm{~min}$ (range: 4-12 $\mathrm{min}$ ) and $13.5 \pm 4.5 \mathrm{~min}$ (range: $8-16 \mathrm{~min}$ ) for the CEUS-CT/MR and CT-CT/MR-MR image fusions, respectively. The results of the AM evaluation based on CEUS-CT/MR and $\mathrm{MR} / \mathrm{MR}$ image fusions are shown in Table 4. An inadequate AM was caused by blood vessels in seven cases (46.7\%) and an inadequate ablation zone in eight cases (53.3\%). Compared with CT-CT/MR-MR image fusion, the sensitivity, specificity, and accuracy of CEUS-CT/MR image fusion for the evaluation of AM were 100.0, 80.0, and $90.0 \%$, respectively.

\section{Discussion}

Phantoms have been widely used to evaluate the effects of thermal treatments. Previous studies, however, have mainly focused on other topics, such as temperature monitoring, energy distribution, the relationship between RF and electrical conductivity, and development of the heating algorithm applied in drug delivery. It is not clear whether phantoms are good models for the evaluation of AM using a CEUS-CT/MR image fusion system. Therefore, in the present study, we established a phantom model to evaluate AM using CEUS-CT/MR image fusion. In our study, we found that the peculiarly

Table 3 The clinical characteristics of the patients and HCC lesions

\begin{tabular}{lc}
\hline Characteristics & Number \\
\hline Gender (Male/Female) & $26 / 0$ \\
Age (mean \pm standard deviation, years) & \\
Virus hepatitis/alcoholic liver disease/no diffuse & $26 / 0 / 0$ \\
hepatic disease & $/$ \\
Liver cirrhosis (yes/no) & $22 / 4$ \\
Tumor number $(1 />1)$ & $19.1 \pm 6.5$ \\
Tumor diameter (mean $\pm S D$, mm) & \\
\hline HCC hepatocellular carcinoma, $M$ median, QR interquartile range &
\end{tabular}


Table 4 The results of AM evaluated by CEUS-CT/MR image fusion and MR/MR image fusion

\begin{tabular}{lllll}
\hline & & \multicolumn{2}{l}{ MR/MR image fusion } & Total \\
\cline { 3 - 4 } & & AM covered & AM not covered & \\
\hline CEUS-CT/MR & AM covered & 15 & 3 & 18 \\
image fusion & AM not covered & 0 & 12 & 12 \\
& Total & 15 & 15 & 30 \\
\hline
\end{tabular}

thermal invertibility and thermal sensitivity of the carrageenan gel were useful for distinguishing the ablative zone, the remaining 'tumor', and the AM after RFA. While the carrageenan hybrid gel used in the present study was not the best material for the evaluation of thermal ablation, especially for temperature variation and energy distribution, we took full advantage of the physical properties of the carrageenan gel. To the best of our knowledge, this is the first report to assess the accuracy of the evaluation of complete RF using an image fusion system that matched pre-RFA and post-RFA images in a tissue-mimicking phantom.

We developed a hybrid gel phantom using carrageenan and other substances, which have a number of important properties, i.e., sufficient strength, low fragility, and low cost. Carrageenan, a high-molecular-weight polysaccharide extracted from red algae, consists of repeating galactose and 3,6-anhydrogalactose units linked by alternating $\alpha-1,3-$ and $\beta-1,4$-glycosidic linkages. Carrageenan can be used in a phantom model because it is inexpensive and safe, as well as broadly applied for the production of gel products and other foods. Additive agents played significant roles in the construction, imaging, and observation by the naked eye. For example, $\mathrm{NaCl}$ was added to the carrageenan gels to adjust the gel conductivity. US contrast agent and iodipin were used to improve the echo or to enhance attenuation. In addition, Congo red, an indicator used for the diagnosis of amyloidosis by generating a bright and distinct red color, was easily distinguished from the opaque gel. The red color may have infiltrated the peripheral gel due to the diffusion of Congo red. However, we believe that Congo red has no influence on the results of the AM evaluation if the whole procedure, including manufacturing, RFA, and assessment of the ablative zone, is completed within $6 \mathrm{~h}$. Using carrageenan together with other substances, we were able to create a large and robust phantom model with excellent shape retention. The turbidity and low fragility of the carrageenan gel in the phantom model ensured accurate image registration. In addition, we designed a phantom that mimicked the tumor lesion (i.e., a visible sphere) to assess the AM using the fusion imaging system after RF. The easy heating and coagulation of the phantom model allowed us to assess the post-RFA destructive zone more accurately. Improved visualization of the target by US and CT, as well as the distinct color of the materials, also improved the ablation assessment. Therefore, the phantom model established herein was successfully used for the evaluation of the AM of the HCC tumor.

The present experimental study results suggest that the US-CT fusion image system can be used to accurately and effectively evaluate AM. However, in one case, US-CT image fusion revealed that the AM was completely covered by the ablative area, and even less than a 1-mm AM was observed in the gross specimen. The false-positive case could be caused by registration error and magnetic positioning system error. Given that the phantom model was idealized to evaluate AM, the feasibility and accuracy was further validated in a clinical assessment.

In our clinical study, the sensitivity, specificity, and accuracy of CEUS-CT/MR image fusion for the evaluation of AM were 100.0, 80.0 and $90.0 \%$, respectively, suggesting that CEUS-CT/MR image fusion is a good tool for evaluating AM after HCC ablation. CEUS-CT/MR image fusion combines the advantages of CEUS and CT/MR and expands the use of both imaging methods, including the high spatial contrast resolution of $\mathrm{CT} / \mathrm{MR}$ and real-time guidance, accessibility, and practicality of ultrasound. In addition, CEUS-CT/MR image fusion greatly improves intraoperative AM evaluation and the localization of tumor lesions compared with MR-MR image fusion.

We discovered three false-positive cases in the clinical study, which might be due to the ability of CEUS to only demonstrate blood perfusion of tissues rather than necrosis. The high temperature of the local zone of ablation may cause swollen tissues and small vessel occlusion, limiting the infiltration of blood into the ablated area. However, occluded small vessels can be reperfused after the local tissue temperature decreases, suggesting that the ablation zone may be over-measured by intraoperative CEUS.

The present study has several limitations. First, the phantom model cannot completely mimic dynamic tissues and organs, such as respiratory movements, which may reduce the accuracy of the registration for image fusion and affect the imaging assessment. While the assessment was performed successfully in the idealized phantom model, some unknown problems may be present in the in vivo experiments, which must be identified and resolved. Second, the phantom models applied in the present study were used for US-CT image fusion, whereas most of the clinical cases were evaluated by CEUS-CT/MR image fusion. Thus, the accuracy of the results may be biased. Third, all of the patients enrolled in this study were male, which may also bias the results. Therefore, further studies with more experience, a larger sampling size and better technology are needed. 


\section{Conclusions}

In conclusion, we successfully established a phantom model for the evaluation of AM using US-CT/MR image fusion. Our results suggest that US-CT/MR image fusion is an accurate approach for evaluating AM after tumor ablation based on both an in vitro model and a clinical study.

\section{Abbreviations}

AM: Ablative margin; CEUS: Contrast-enhanced ultrasound; HCC: Hepatocellular carcinoma; LTP: Local tumor progression; RFA: Radiofrequency ablation

\section{Acknowledgements}

The authors thank all participating clinicians and general practitioners.

\section{Funding}

This work is supported by the National Natural Science Foundation of China. $\mathrm{RZ}$ is funded by Research Cooperation Project of Guangdong Province. $\mathrm{KL}$ and EX are scholars of Science and Technology Planning Project of Guangdong Province.

\section{Availability of data and materials}

The datasets supporting the conclusions of this article are presented in the main manuscript.

\section{Authors' contribution}

RZ contributed to the conception of the study. $\mathrm{KL}$ and ZS performed the experiments. QH and QZ were responsible for gathering data. EX and $\mathrm{KL}$ analyzed the data. $\mathrm{KL}$ wrote the manuscript. All authors read and approved the final manuscript.

\section{Competing interests}

The authors declare that they have no competing interests.

\section{Consent for publication}

\section{Not applicable.}

\section{Ethics approval and consent to participate}

This study was approved by the Institute Research Medical Ethics Committee of the Third Affiliated Hospital of Sun Yat-Sen University and was in compliance with the Declaration of Helsinki. Informed consent was obtained from all participants. All subjects signed informed consents prior to their inclusion in the study.

Received: 18 August 2016 Accepted: 12 January 2017

Published online: 19 January 2017

\section{References}

1. Bruix J, Han KH, Gores G, Llovet JM, Mazzaferro V. Liver cancer: Approaching a personalized care. J Hepatol. 2015;62:S144-56.

2. Kim YS, Lim HK, Rhim H, Lee MW. Ablation of hepatocellular carcinoma. Best Pract Res Clin Gastroenterol. 2014;28:897-908.

3. Huang J, Yan L, Cheng Z, Wu H, Du L, Wang J, et al. A randomized trial comparing radiofrequency ablation and surgical resection for HCC conforming to the Milan criteria. Ann Surg. 2010;252:903-12.

4. Liu Z, Zhou Y, Zhang P, Qin H. Meta-analysis of the therapeutic effect of hepatectomy versus radiofrequency ablation for the treatment of hepatocellular carcinoma. Surgical Laparoscopy Endoscopy \& Percutaneous Techniques. 2010;20:130-40.

5. Tiong L, Maddern GJ. Systematic review and meta-analysis of survival and disease recurrence after radiofrequency ablation for hepatocellular carcinoma. Br J Surg. 2011;98:1210-24.

6. Yi HM, Zhang W, Ai X, Li KY, Deng YB. Radiofrequency ablation versus surgical resection for the treatment of hepatocellular carcinoma conforming to the Milan criteria: systemic review and meta-analysis. Int J Clin Exp Med. 2014;7:3150-63.

7. Ayav A, Germain A, Marchal F, Tierris I, Laurent V, Bazin C, et al. Radiofrequency ablation of unresectable liver tumors: factors associated with incomplete ablation or local recurrence. Am J Surg. 2010;200:435-9.
8. Hirooka M, Ochi H, Koizumi Y, Tokumoto Y, Hiraoka A, Kumagi T, et al. Local recurrence of hepatocellular carcinoma in the tumor blood drainage area following radiofrequency ablation. Mol Clin Oncol. 2014;2:182-6.

9. Kei SK, Rhim H, Choi D, Lee WJ, Lim HK, Kim YS. Local tumor progression after radiofrequency ablation of liver tumors: analysis of morphologic pattern and site of recurrence. AJR Am J Roentgenol. 2008;190:1544-51.

10. Komorizono Y, Oketani M, Sako K, Yamasaki N, Shibatou T, Maeda M, et al. Risk factors for local recurrence of small hepatocellular carcinoma tumors after a single session, single application of percutaneous radiofrequency ablation. Cancer. 2003;97:1253-62.

11. Lee HY, Rhim H, Lee MW, Kim YS, Choi D, Park MJ, et al. Early diffuse recurrence of hepatocellular carcinoma after percutaneous radiofrequency ablation: analysis of risk factors. Eur Radiol. 2013;23:190-7.

12. Liu CH, Arellano RS, Uppot RN, Samir AE, Gervais DA, Mueller PR. Radiofrequency ablation of hepatic tumours: effect of post-ablation margin on local tumour progression. Eur Radiol. 2010;20:877-85.

13. Nishikawa H, Inuzuka T, Takeda H, Nakajima J, Sakamoto A, Henmi S, et al. Percutaneous radiofrequency ablation therapy for hepatocellular carcinoma: a proposed new grading system for the ablative margin and prediction of local tumor progression and its validation. J Gastroenterol. 2011;46:1418-26.

14. Teng W, Liu KW, Lin CC, Jeng WJ, Chen WT, Sheen IS, et al. Insufficient ablative margin determined by early computed tomography may predict the recurrence of hepatocellular carcinoma after radiofrequency ablation. Liver Cancer. 2015;4:26-38.

15. Yu J, Liang P, Yu XL, Cheng ZG, Han ZY, Mu MJ, et al. Local tumour progression after ultrasound-guided microwave ablation of liver malignancies: risk factors analysis of 2529 tumours. Eur Radiol. 2015;25:1119-26.

16. Ahmed M, Solbiati L, Brace CL, Breen DJ, Callstrom MR, Charboneau JW, et al. Image-guided tumor ablation: standardization of terminology and reporting criteria-a 10-year update. Radiology. 2014;273:241-60.

17. Dodd 3rd GD, Frank MS, Aribandi M, Chopra S, Chintapalli KN. Radiofrequency thermal ablation: computer analysis of the size of the thermal injury created by overlapping ablations. AJR Am J Roentgenol. 2001;177:777-82.

18. Li K, Su ZZ, Xu EJ, Ju JX, Meng XC, Zheng RQ. Improvement of ablative margins by the intraoperative use of CEUS-CT/MR image fusion in hepatocellular carcinoma. BMC Cancer. 2016;16:277.

19. Nakazawa T, Kokubu S, Shibuya A, Ono K, Watanabe M, Hidaka H, et al. Radiofrequency ablation of hepatocellular carcinoma: correlation between local tumor progression after ablation and ablative margin. AJR Am J Roentgenol. 2007;188:480-8.

20. Zytoon AA, Ishii H, Murakami K, El-Kholy MR, Furuse J, El-Dorry A, et al. Recurrence-free survival after radiofrequency ablation of hepatocellular carcinoma. A registry report of the impact of risk factors on outcome. Jpn J Clin Oncol. 2007;37:658-72.

21. Kim YS, Lee WJ, Rhim H, Lim HK, Choi D, Lee JY. The minimal ablative margin of radiofrequency ablation of hepatocellular carcinoma ( $>2$ and $<5 \mathrm{~cm}$ ) needed to prevent local tumor progression: 3D quantitative assessment using CT image fusion. AJR Am J Roentgenol. 2010;195:758-65.

22. Makino $Y$, Imai $Y$, Igura $T$, Hori M, Fukuda $K$, Sawai $Y$, et al. Utility of computed tomography fusion imaging for the evaluation of the ablative margin of radiofrequency ablation for hepatocellular carcinoma and the correlation to local tumor progression. Hepatol Res. 2013;43:950-8.

23. Sakakibara M, Ohkawa K, Katayama K, Imanaka K, Ishihara A, Hasegawa N, et al. Three-dimensional registration of images obtained before and after radiofrequency ablation of hepatocellular carcinoma to assess treatment adequacy. AJR Am J Roentgenol. 2014;202:W487-95.

24. Wang XL, Li K, Su ZZ, Huang ZP, Wang P, Zheng RQ. Assessment of radiofrequency ablation margin by MRI-MRI image fusion in hepatocellular carcinoma. World J Gastroenterol. 2015;21:5345-51.

25. Zhong-Zhen S, Kai L, Rong-Qin Z, Er-Jiao X, Ting Z, Ao-Hua Z, et al. A feasibility study for determining ablative margin with 3D-CEUS-CT/MR image fusion after radiofrequency ablation of hepatocellular carcinoma. Ultraschall Med. 2012;33:E250-5.

26. Mori K, Fukuda K, Asaoka H, Ueda T, Kunimatsu A, Okamoto Y, et al. Radiofrequency ablation of the liver: determination of ablative margin at MR imaging with impaired clearance of ferucarbotran-feasibility study. Radiology. 2009;251:557-65.

27. Tokunaga S, Koda M, Matono T, Sugihara T, Nagahara T, Ueki M, et al. Assessment of ablative margin by MRI with ferucarbotran in radiofrequency ablation for liver cancer: comparison with enhanced CT. Br J Radiol. 2012;85:745-52. 
28. Okubo H, Kokubu S, Komiyama M, Yamagata H, Uchiyama A, Miyazaki A, et al. Radiofrequency ablation of hepatocellular carcinoma: The feasibility of magnetic resonance imaging with gadolinium ethoxybenzyl diethylene triamine pentaacetic acid for evaluating the ablative margin. Hepatol Res. 2010;40:1034-41.

29. Bruix J, Sherman M. Management of hepatocellular carcinoma: an update. Hepatology. 2011;53:1020-2.

Submit your next manuscript to BioMed Central and we will help you at every step:

- We accept pre-submission inquiries

- Our selector tool helps you to find the most relevant journal

- We provide round the clock customer support

- Convenient online submission

- Thorough peer review

- Inclusion in PubMed and all major indexing services

- Maximum visibility for your research

Submit your manuscript at www.biomedcentral.com/submit
Biomed Central 